\title{
OPEN Application of evoked response audiometry for specifying aberrant gamma oscillations in schizophrenia
}

\begin{abstract}
Masaya Yanagi $^{1 凶}$, Aki Tsuchiya ${ }^{1}$, Fumiharu Hosomi ${ }^{1}$, Satoshi Ozaki ${ }^{2}$ \& Osamu Shirakawa ${ }^{1}$
Gamma oscillations probed using auditory steady-state response (ASSR) are promising clinical biomarkers that may give rise to novel therapeutic interventions for schizophrenia. Optimizing clinical settings for these biomarker-driven interventions will require a quick and easy assessment system for gamma oscillations in psychiatry. ASSR has been used in clinical otolaryngology for evoked response audiometry (ERA) in order to judge hearing loss by focusing on the phase-locked response detectability via an automated analysis system. Herein, a standard ERA system with $40-$ and $46-\mathrm{Hz}$ ASSRs was applied to evaluate the brain pathophysiology of patients with schizophrenia. Both ASSRs in the ERA system showed excellent detectability regarding the phase-locked response in healthy subjects and sharply captured the deficits of the phase-locked response caused by aberrant gamma oscillations in individuals with schizophrenia. These findings demonstrate the capability of the ERA system to specify patients who have aberrant gamma oscillations. The ERA system may have a potential to serve as a real-world clinical medium for upcoming biomarker-driven therapeutics in psychiatry.
\end{abstract}

Gamma oscillations whose impairments are predominantly shown in the prefrontal cortex are promising clinical biomarkers that may address novel therapeutic interventions for schizophrenia ${ }^{1-7}$. These are neural, rhythmic fluctuations in the gamma frequency range $(30-200 \mathrm{~Hz})$ that are commonly captured by electroencephalogram (EEG) or magnetoencephalography ${ }^{2,8}$. Gamma oscillations play a role in information processing in higher-order brain functions (e.g., perception, attention, and working memory). Hence, various types of sensory and cognitive stimuli can elicit gamma oscillations in the associated brain regions $\mathrm{s}^{2,6,8}$. The auditory steady-state response (ASSR) is a sustained neural entrainment to periodic auditory stimuli, which can probe the ability to generate gamma oscillations by temporally modulating the stimuli at gamma frequency ranges. ASSR potentials in humans are largest when the periodic auditory stimuli are presented at a frequency of approximately $40 \mathrm{~Hz}^{9}$.

Previous ASSR studies have shown that the $40-\mathrm{Hz}$ gamma oscillations are highly impaired in patients with schizophrenia ${ }^{10}$. The impaired $40-\mathrm{Hz}$ gamma oscillations implicate abnormal functional interaction between parvalbumin-positive GABAergic neurons and pyramidal neurons in the prefrontal cortex of schizophrenia patients $^{11-13}$. Considering the evidence, provided by rodent studies, that the cortical parvalbumin-positive GABAergic neurons are the generators of gamma oscillations ${ }^{14,15}$, the parvalbumin-positive GABAergic neurons could be the primary target in the treatment of impaired gamma oscillations ${ }^{16}$. Based on the findings of postmortem brain studies, the cortical parvalbumin-positive GABAergic neurons are impaired in the prefrontal cortex of patients with schizophrenia ${ }^{1,17}$, and GABAergic compounds are challenged to compensate for the dysfunction of the GABAergic neurons in schizophrenia patients ${ }^{18-20}$. Kv3.1 is a potassium channel involved in the firing of parvalbumin-positive GABAergic neurons ${ }^{21}$. A compound for modulating Kv3.1 activity has been developed to treat schizophrenia ${ }^{22}$ based on postmortem findings of the brain, the prefrontal reduction of this channel protein in schizophrenia patients ${ }^{23}$. However, given the heterogeneous nature of schizophrenia, the therapeutic targets of such compounds may be optimal for some, but not all, schizophrenia patients ${ }^{24,25}$. Given that gamma oscillations have the potential to detect such targeting pathophysiology among patients with schizophrenia, a simple system to assess the gamma oscillations will be required in the future in psychiatry practice.

ASSR is currently used in clinical otolaryngology for evoked response audiometry (ERA) with medically approved devices. The ASSR in the ERA system is performed by means of an automated analysis that is designed

${ }^{1}$ Department of Neuropsychiatry, Faculty of Medicine, Kindai University, 377-2 Ohnohigashi, Osaka-sayama, Osaka 589-8511, Japan. ${ }^{2}$ Izumigaoka Hospital, Izumi, Osaka, Japan. ${ }^{\circledR}$ email: yanagi@med.kindai.ac.jp 


\section{a. Phase-locked response}

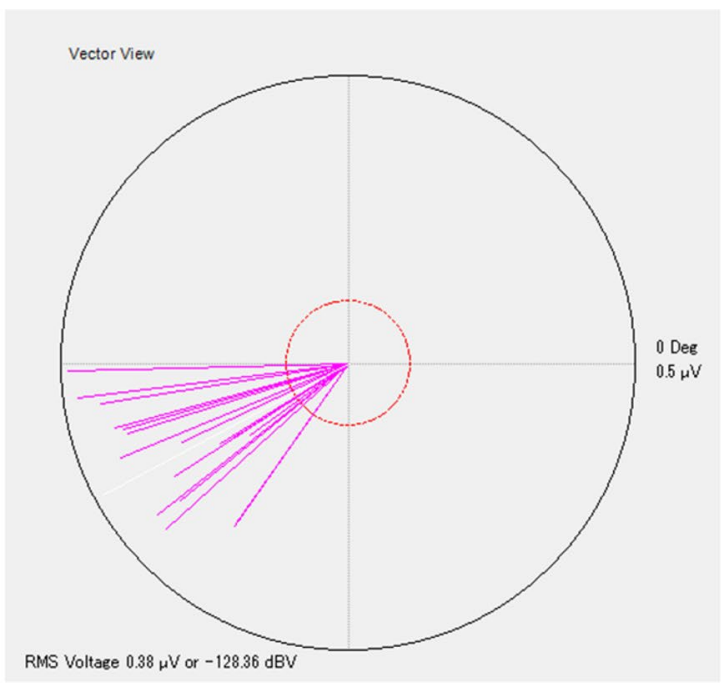

\section{b. Non-phase-locked response}

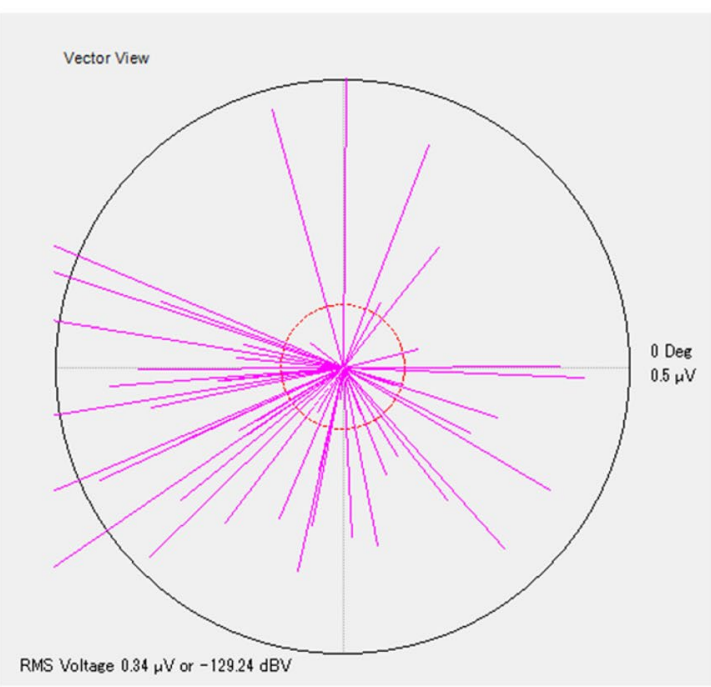

Figure 1. The representative phase-locked (a) and non-phase-locked (b) responses in 46-Hz AM-FM ASSR in the ERA system. (a) and (b) are cases of a healthy participant and a patient with schizophrenia, respectively. Each vector in the diagram, which was created by each trial during the auditory stimulus, represents the phase and the amplitude of EEG activity corresponding to the tone modulation frequency rate. The phase angle of the vectors corresponds to the time delay between the presentation of the stimulus and the neural response of trials. The cross-trial phase consistency of the vectors determines the phase-locked or non-phase-locked response. While all trials are aligned in the same range in (a), the nonaligned trials that prevent the identification of phaselocked response are seen in (b). The mean length of the vectors represents the response amplitude (root mean square voltage, in microvolts) of the ASSR.

\begin{tabular}{|c|c|c|c|c|c|c|}
\hline \multirow[b]{2}{*}{ ASSR } & & \multicolumn{4}{|c|}{$\begin{array}{l}\text { Occurrence of } \\
\text { responses for phase- } \\
\text { locked/non-phase- } \\
\text { locked }\end{array}$} & \multirow[b]{2}{*}{ Fisher's exact test } \\
\hline & & $3 / 0$ & $2 / 1$ & $1 / 2$ & $0 / 3$ & \\
\hline \multirow{2}{*}{ 46-Hz AM-FM } & Healthy subjects, $\mathrm{n}=38$ & 38 & 0 & 0 & 0 & \multirow{2}{*}{$<0.0001$} \\
\hline & Patients with schizophrenia, $\mathrm{n}=38$ & 24 & 5 & 5 & 4 & \\
\hline \multirow{2}{*}{$40-\mathrm{Hz} \mathrm{AM}$} & Healthy subjects & 38 & 0 & 0 & 0 & \multirow{2}{*}{0.001} \\
\hline & Patients with schizophrenia & 28 & 3 & 4 & 3 & \\
\hline
\end{tabular}

Table 1. Occurrence of the phase-locked and non-phase-locked responses in thrice measurements of $40-\mathrm{Hz}$ ASSR.

to judge hearing loss by focusing on the phase-locked response detectability. These devices are globally available at many hospital facilities, and the established clinical protocol for measurements ensures the reproducibility of the ASSR results. Furthermore, the $40-\mathrm{Hz}$ ASSR is the testing condition in ERA for awake adults, and this configuration is preset in the devices with a stimulus rate of around $40 \mathrm{~Hz}$. In the standard ERA device, Audera (Grason-Stadler Inc., Eden Prairie, MN, USA), this test is set as an ASSR by the stimulation of a $46-\mathrm{Hz}$ amplitude modulation (AM) tone with slight frequency modulation (FM). That is, the test was set as a 46-Hz AM-FM ASSR. To utilize the plausible availability of the ERA system, this study examined the detectability of impaired gamma oscillations in patients with schizophrenia using the ERA device with the 46-Hz AM-FM ASSR as well as an ASSR with a basic 40-Hz AM tone stimulation, (i.e., 40-Hz AM ASSR).

\section{Results}

Phase-locked/non-phase-locked response. Figure 1 shows the phase-locked (A) and non-phaselocked (B) representative responses in 46-Hz AM-FM ASSR. All of the measurement conducted thrice in the healthy subjects $(n=38)$ demonstrated a phase-locked response in both $46-\mathrm{Hz}$ AM-FM ASSR and 40-Hz AM ASSR. Conversely, a non-phase-locked response was demonstrated in 14 patients in 46-Hz AM-FM ASSR and 10 patients in $40-\mathrm{Hz}$ AM ASSR among the 38 patients with schizophrenia. The details of the occurrence of the phase- and non-phase-locked responses are provided in Table 1, and the results for each patient are in the Supplementary Table. All patients who showed non-phase-locked responses in 40-Hz AM ASSR also showed nonphase-locked responses in 46-Hz AM-FM ASSR, whereas some patients showed non-phase-locked responses 


\begin{tabular}{|c|c|c|c|}
\hline & All phase-locked ${ }^{a}$ & Non-phase-locked ${ }^{b}$ & $P$ value \\
\hline \multicolumn{4}{|l|}{ 46-Hz AM-FM ASSR } \\
\hline Number of cases & 24 & 14 & \\
\hline Gender (male/female) & $12 / 12$ & $8 / 6$ & 0.61 \\
\hline Age, $y$, mean \pm SD & $44.0 \pm 11.1$ & $51.4 \pm 9.1$ & 0.03 \\
\hline Illness duration, $\mathrm{y}$ & $20.3 \pm 11.8$ & $27.1 \pm 11.4$ & 0.73 \\
\hline GAF & $35.9 \pm 15.5$ & $31.1 \pm 17.2$ & 0.12 \\
\hline \multicolumn{4}{|l|}{ BPRS, four-dimensional model } \\
\hline Thinking disturbance $^{\mathrm{c}}$ & $5.0 \pm 3.3$ & $8.1 \pm 4.8$ & 0.20 \\
\hline Withdrawal/retardation $^{\mathrm{d}}$ & $4.5 \pm 3.4$ & $5.9 \pm 4.9$ & 0.27 \\
\hline Hostile/suspiciousness & $2.6 \pm 2.9$ & $3.0 \pm 2.9$ & 0.06 \\
\hline Anxious/depression & $2.7 \pm 2.3$ & $3.2 \pm 2.4$ & 0.07 \\
\hline Antipsychotics ${ }^{\mathrm{e}}, \mathrm{mg} /$ day & $700 \pm 99$ & $1388 \pm 336$ & 0.06 \\
\hline Classification of antipsychotics ${ }^{\mathrm{f}}$ & $12 / 12$ & $3 / 11$ & 0.06 \\
\hline \multicolumn{4}{|l|}{ 40-Hz AM ASSR } \\
\hline Number of cases & 28 & 10 & \\
\hline Gender (male/female) & $16 / 12$ & $4 / 6$ & 0.83 \\
\hline Age, $y$, mean \pm SD & $45.0 \pm 11.3$ & $51.5 \pm 8.1$ & 0.05 \\
\hline Illness duration, $\mathrm{y}$ & $21.3 \pm 12.3$ & $27.0 \pm 10.7$ & 0.69 \\
\hline GAF & $35.0 \pm 16.1$ & $31.6 \pm 16.4$ & 0.14 \\
\hline \multicolumn{4}{|l|}{ BPRS, four-dimensional model } \\
\hline Thinking disturbance & $5.4 \pm 3.2$ & $8.3 \pm 5.8$ & 0.11 \\
\hline Withdrawal/retardation & $4.5 \pm 3.3$ & $6.7 \pm 5.3$ & 0.10 \\
\hline Hostile/suspiciousness & $2.8 \pm 3.1$ & $2.6 \pm 2.3$ & 0.12 \\
\hline Anxiety/depression & $2.9 \pm 2.3$ & $2.8 \pm 2.3$ & 0.18 \\
\hline Antipsychotics, mg/day & $901 \pm 183$ & $1099 \pm 223$ & 0.75 \\
\hline Classification of antipsychotics & $13 / 15$ & $2 / 8$ & 0.05 \\
\hline
\end{tabular}

Table 2. Comparison of clinical variables between all phase-locked and non-phase-locked groups in

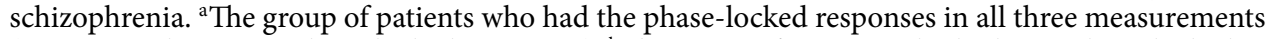

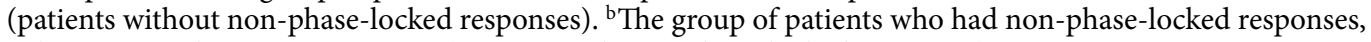

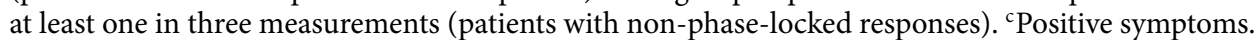
${ }^{\mathrm{d}}$ Negative symptoms. ${ }^{\mathrm{e}} \mathrm{Chlorpromazine} \mathrm{equivalent} \mathrm{dose.}{ }^{\mathrm{f}} \mathrm{New}$ generation antipsychotics only/Conventional antipsychotics or combination (new generation and conventional antipsychotics).

only in 46-Hz AM-FM ASSR. The difference between the two ASSRs in terms of the number of patients with non-phase-locked responses was not significant $(P=0.46$, Fisher's exact test).

Clinical variables in patients with and without non-phase-locked responses. We defined the all phase-locked group as those who manifested phase-locked responses in all three ASSR measurements and the non-phase-locked group as the patients who manifested at least one non-phase-locked response in the three ASSR measurements. In the logistic regression analysis, significant differences were found between the two groups for both ASSRs in terms of age and in terms of classification of antipsychotics for the 40-Hz AM ASSR (Table 2). No other significant differences were found in terms of other clinical variables between the two groups in either the 46-Hz AM-FM ASSR or the 40-Hz AM ASSR.

Number of trials to achieve phase-locked response. To further investigate the details of ASSR, additional parameters were examined. Significant increases were noted in the number of trials required to achieve a phase-locked response in patients with schizophrenia (46-Hz AM-FM ASSR, mean $\pm \mathrm{SD}=35.5 \pm 17.4 ; 40-\mathrm{Hz}$ AM ASSR, 31.0 \pm 15.9 ) as compared with healthy subjects (46-Hz AM-FM ASSR, 20.2 $\pm 3.8 ; 40-\mathrm{Hz}$ AM ASSR, $20.3 \pm 3.3$ ) in either of the ASSRs (46-Hz AM-FM ASSR, $t=5.3, d f=74, P<0.0001 ; 40-\mathrm{Hz}$ AM ASSR, $t=4.0$, $d f=74, P=0.0001$ ) (Fig. 2a).

Response amplitude. The response amplitude of the 40-Hz ASSR did not significantly differ between patients with schizophrenia (46-Hz AM-FM ASSR, $0.35 \pm 0.26 \mu \mathrm{V} ; 40-\mathrm{Hz}$ AM ASSR, $0.35 \pm 0.16 \mu \mathrm{V}$ ) and healthy subjects (46-Hz AM-FM ASSR, $0.38 \pm 0.12 \mu \mathrm{V} ; 40-\mathrm{Hz}$ AM ASSR, $0.36 \pm 0.11 \mu \mathrm{V})$ in either of the ASSRs $(46-\mathrm{Hz}$ AM-FM ASSR, $t=0.72, d f=74, P=0.47 ; 40-\mathrm{Hz}$ AM ASSR, $t=0.43, d f=74, P=0.67$ ). These results are depicted in Fig. 2b. In addition, no significant differences were observed between patients with non-phase-locked responses (46-Hz AM-FM ASSR, $0.34 \pm 0.36 \mu \mathrm{V} ; 40-\mathrm{Hz}$ AM ASSR, $0.34 \pm 0.18 \mu \mathrm{V}$ ) and those without non-phase-locked 
a Number of trials to achieve phase-locked response

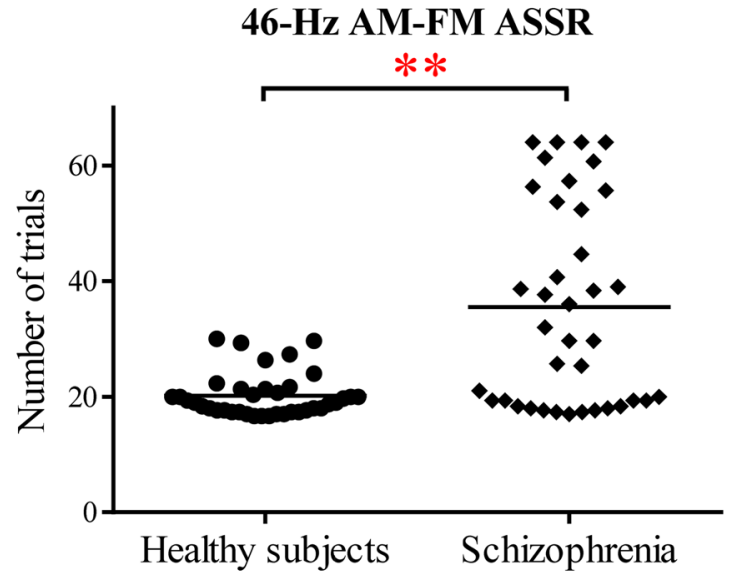

b Response amplitude

\section{6-Hz AM-FM ASSR}

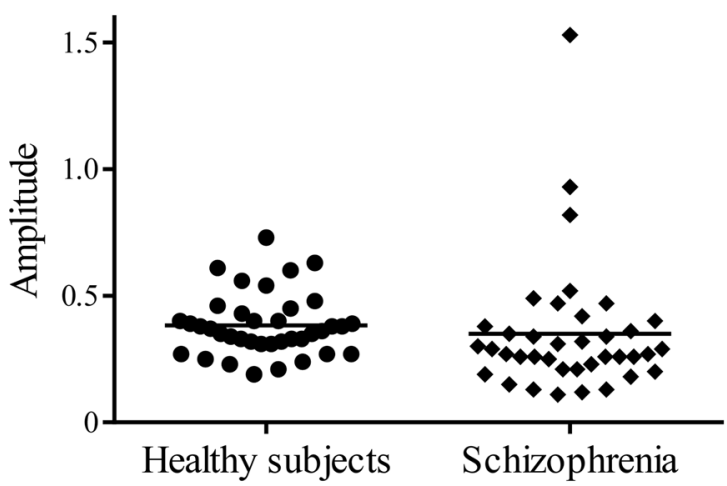

40-Hz AM ASSR

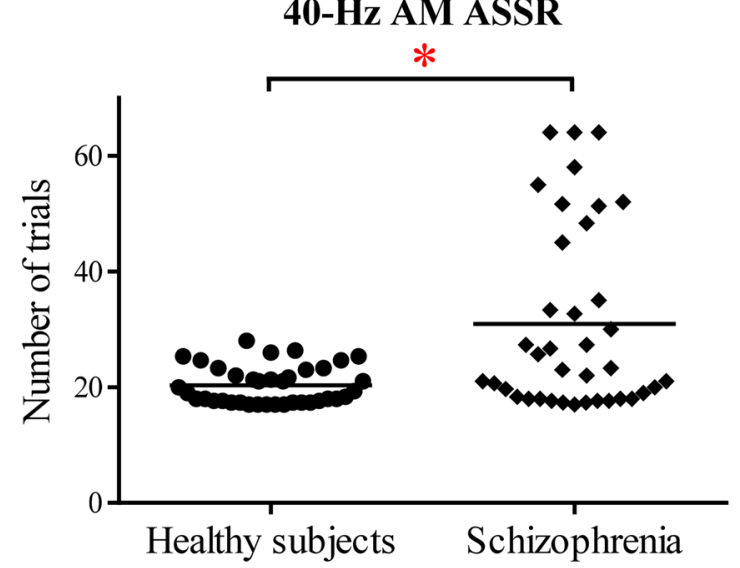

40-Hz AM ASSR

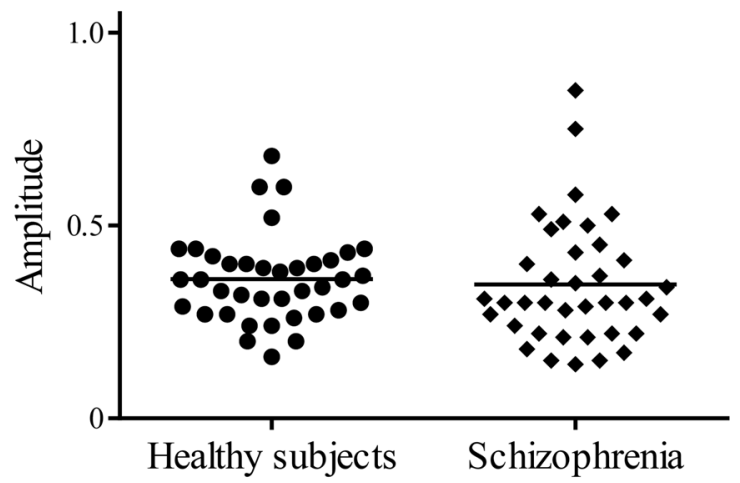

Figure 2. Trends to achieve the phase-locked response and the ASSR potentials. (a) Significant differences were observed in the number of trials conducted to achieve a phase-locked response between patients with schizophrenia and healthy subjects in 46-Hz AM-FM ASSR (left) and 40-Hz AM ASSR (right) ${ }^{* \star P} P<0.0001$, ${ }^{\star} P=0.0001$. (b) No significant differences were observed between patients with schizophrenia and healthy subjects in terms of the response amplitudes of 46-Hz AM-FM ASSR (left) and 40-Hz AM ASSR (right).

\begin{tabular}{|l|l|l|l|l|}
\hline \multirow{2}{*}{} & \multicolumn{2}{|l|}{$\begin{array}{l}\text { Number of trials to achieve the phase- } \\
\text { locked response }\end{array}$} & \multicolumn{2}{l|}{ Response amplitude } \\
\cline { 2 - 5 } & $\mathbf{4 6 - H z}$ AM-FM ASSR & $\mathbf{4 0 - H z}$ AM ASSR & 46-Hz AM-FM ASSR & 40-Hz AM ASSR \\
\hline \multirow{2}{*}{ Average-measure ICC 95\% CI } & $0.92^{\mathrm{a}}$ & $0.89^{\mathrm{a}}$ & $0.94^{\mathrm{a}}$ & $0.92^{\mathrm{a}}$ \\
\cline { 2 - 5 } & $0.88-0.95$ & $0.83-0.92$ & $0.92-0.96$ & $0.88-0.94$ \\
\hline \multirow{2}{*}{ Single-measure ICC 95\% CI } & $0.79^{\mathrm{b}}$ & $0.72^{\mathrm{b}}$ & $0.85^{\mathrm{a}}$ & $0.78^{\mathrm{b}}$ \\
\cline { 2 - 5 } & $0.71-0.85$ & $0.62-0.80$ & $0.79-0.90$ & $0.70-0.85$ \\
\hline
\end{tabular}

Table 3. Test-retest reliabilities by intraclass correlation coefficient (ICC) among thrice measurements of

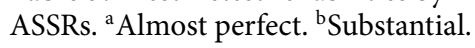

responses (46-Hz AM-FM ASSR, $0.35 \pm 0.19 \mu \mathrm{V} ; 40-\mathrm{Hz}$ AM ASSR, $0.35 \pm 0.15 \mu \mathrm{V})$ in either of the ASSRs $(46-\mathrm{Hz}$ AM-FM ASSR, $t=0.08, d f=36, P=0.94 ; 40-\mathrm{Hz}$ AM ASSR, $t=0.28, d f=36, P=0.78$ ).

Test-retest reliabilities of the ASSR measurements. The number of trials to achieve the phaselocked response and the response amplitude showed consistent results among the three measurements (as shown in Fig. S1) for the entire sample of patients and healthy subjects. Table 3 shows the ICCs among the three ASSR measurements. The average-measure intraclass correlation coefficients (ICCs) showed almost perfect reli- 
abilities for both ASSRs in regard to both the number of trials required to achieve a phase-locked response and the response amplitude (Table 3). The single-measure ICCs showed substantial reliabilities for all conditions except for the response amplitude in 46-Hz AM-FM ASSR, which showed almost perfect reliability (Table 3).

\section{Discussion}

The current study proposes an approach that uses an automated ERA system to specify individual patients with schizophrenia who have severely impaired $40-\mathrm{Hz}$ gamma oscillations. These findings may serve to address the biological variability in schizophrenia patients as it relates to upcoming biomarker-driven therapeutics. The ERA system, which showed excellent detectability of the phase-locked response in healthy subjects, sharply captured the non-phase-locked responses in individuals with schizophrenia in both 46-Hz AM-FM ASSR and $40-\mathrm{Hz}$ AM ASSR (Table 1). Almost perfect test-retest reliabilities were shown among the three measurements of each ASSR in its average-measure ICC for the number of trials required to achieve a phase-locked response, which is the index by which phase-locked response is judged (Table 3). This reproducibility of the repeated ASSR measurements via automatic analysis supports the potential of the ERA system in clinical settings to specify the patients who have severe impairments, which present as non-phase-locked responses in $40-\mathrm{Hz}$ ASSR. Furthermore, the advanced utility of the ERA system in the real-world clinical setting suggests its potential utility in real-world clinical applications in psychiatry. The global ERA system is suitable for use in extensive clinical trials to recruit patients compatible with novel treatments to improve $40-\mathrm{Hz}$ gamma oscillation abnormalities. These biomarker-driven treatments can then be expanded into clinical practices, in which the established clinical procedures of ASSR ensure the reproducibility of results across facilities, globally. Thus, the ERA system could significantly enhance the clinical applications of $40-\mathrm{Hz}$ ASSR due to its simple and globally advanced utility regarding the development of novel and targeted treatments to ameliorate impaired gamma oscillations in schizophrenia patients.

Further clinical investigation into these ASSRs between the all phase-locked and non-phase-locked groups in patients with schizophrenia revealed significant differences in age across both ASSRs as well as in the classification of antipsychotics in the 40-Hz AM ASSR (Table 2). A similar trend was found in the classification of antipsychotics in the 46- Hz AM-FM ASSR (Table 2). Although these findings need to be confirmed in larger cohorts before a conclusion can be drawn, a previous study reported that $40-\mathrm{Hz}$ ASSR was enhanced in patients with new generation antipsychotics as compared to conventional antipsychotics ${ }^{26}$. These findings suggest that the further development of new generation antipsychotics whose effects go beyond that of a dopaminergic blockade may ameliorate the impaired $40-\mathrm{Hz}$ ASSR in patients with schizophrenia.

The results of the current study showed that non-phase-locked responses were observed more frequently in 46- Hz AM-FM ASSR as compared with 40-Hz AM ASSR in schizophrenia patients although the difference between the two ASSRs in terms of these frequencies was not statistically significant. A major difference between the auditory stimuli of 46-Hz AM-FM ASSR and 40-Hz AM ASSR is the fluctuation of the tone induced by the FM. Although other differences in the modulated rate $(46 \mathrm{~Hz}$ vs. $40 \mathrm{~Hz})$ exist between the two stimuli, a previous study exhibited similar abnormalities in ASSR between the 40 - and $45-\mathrm{Hz}$ rates in patients with schizophrenia $^{27}$. Therefore, we speculate that the complex tone stimulation in which the FM component was added to the AM is more sensitive than the AM-only treatment in regard to the neural entrainment dysfunction in schizophrenia patients. FM detection is generally crucial for speech perception, especially under noisy conditions (e.g., the presence of competing voices) ${ }^{28}$, and abnormal speech perception has been reported in patients with schizophrenia ${ }^{29,30}$. The FM variation in the $40-\mathrm{Hz}$ ASSR may be worth exploring in further studies in order to develop an understanding of the abnormal speech perception in schizophrenia patients.

Accumulating evidence has shown that, in addition to the reduced phase-locking, the evoked power that quantifies the phase-locked activity in the $40-\mathrm{Hz}$ ASSR is reduced in patients with schizophrenia ${ }^{10}$. The ERA system does not provide the results to examine the evoked power, though it does provide averaged response amplitude that quantifies both phase-locked and non-phase-locked activities together. In both the 46-Hz AM-FM ASSR and the $40-\mathrm{Hz}$ AM ASSR trials, the response amplitudes did not differ significantly between patients with schizophrenia and healthy subjects (Fig. 2b) despite patients having poor phase-locked responses (Fig. 2a). The typical reason for this phenomenon is represented in Fig. 1. The ASSR with the non-phase-locked response consisted of abundant non-phase-aligned trials that prevented the identification of a phase-locked response (Fig. 1b). These non-phase-aligned activities potently occur at similar amplitude levels to those of normal phase-locked activities (Fig. 1a). Previous studies reported that patients with schizophrenia have aberrant gamma oscillations that are heightened induced (stimulus-induced non-phase-locked) gamma activities that are accompanied by a reduced phase-locking of $40-\mathrm{Hz}$ ASSR $^{31,32}$. These aberrant gamma oscillations may be linked with those shown as the prevailing non-phase-aligned measurements during the ASSR trials in this study.

This is an initial study of the ERA system to examine impaired $40-\mathrm{Hz}$ gamma oscillations in schizophrenia patients. Further development of this assessment system is required to optimize into clinical settings in psychiatry. First, the Audera system defines the non-phase-locked response as cases that did not reach a phase-locked response within 64 trials. This cutoff criterion to define a non-phase-locked response needs to be refined as clinical tests with the ERA system progress in psychiatry. However, the cutoff criterion ought not to be much less than 40 trials so as to avoid including the few impairments of 40-Hz ASSR that can be seen even in the healthy subjects in our study (Fig. 2a). Second, further development of the ERA device software is required. The ERA system provides only single summary results for the frequency domain activity, and it does not store the EEG recordings needed for a detailed analysis of the ASSR. Therefore, we cannot compare the results from the ERA system with commonly used parameters such as the evoked power or the phase-locking factor (PLF). This is a limitation of the current ERA system in regard to a comprehensive understanding of the pathophysiology encircling the impaired $40-\mathrm{Hz}$ ASSR in schizophrenia patients. Third, the influence of the methodological difference between the ERA 
system and the methods employed in previous ASSR studies in schizophrenia needs to be characterized. The ERA system uses continuous auditory tone stimuli, while previous studies for schizophrenia have used discrete stimuli. A time-frequency decomposition analysis, which is currently the standard analytical method in schizophrenia research ${ }^{33}$, has developed with the discrete tone stimuli. This analysis provides not only basic results, such as PLF and evoked power ${ }^{33}$ but also collateral information such as transient onset/offset responses ${ }^{34}$ and the time course of the response to the discrete tones ${ }^{35-38}$. Nonetheless, the continuous tone stimuli have an advantage that can shorten measurement time, which helps the examinee to avoid falling asleep, a factor confounded with decreasing the amplitude of $40-\mathrm{Hz}$ ASSR $^{39}$. Another ingenuity of the ERA system is the use of short epochs for overlay, which can easily enhance the overlay of epochs up to hundreds of times (up to 640 times in Audera) in a short period. This is favorable for reliably detecting the non-phase-locked response. Further studies are needed to integrate the advantages of the ERA system into schizophrenia research, and such integration might diminish the inconsistency of the findings associated with 40-Hz ASSR in studies on schizophrenia.

In this study, we demonstrated the capability of the ERA system to specify aberrant $40-\mathrm{Hz}$ gamma oscillations in patients with schizophrenia. Given that schizophrenia is a heterogeneous disease whose pathophysiology is shared with other psychiatric diseases, such as bipolar disorder ${ }^{25,34,40}$, the ERA system may be used adjunctively with other biomarkers to biologically classify schizophrenia and related disorders by seeking optimal therapeutic targets with $40-\mathrm{Hz}$ ASSR. Further clinical studies with $40-\mathrm{Hz}$ ASSR via an ERA system are warranted so as to inform the construction of a solid biomarker for the target treatment in the concept of the biological classifications of schizophrenia and related disorders.

\section{Methods}

Subjects. This study recruited 38 patients with schizophrenia (20 males and 18 females) aged between 24 and 67 years old (mean $\pm S D=46.2 \pm 11.2)$ and 38 case-matched healthy subjects ( 20 males and 18 females) aged between 27 and 68 years $(46.7 \pm 10.9)$ from Kindai University Hospital and Izumigaoka Hospital. Each patient was diagnosed based on the DSM- 5 criteria $^{41}$. Clinical symptoms and social functioning were assessed using the Brief Psychiatric Rating Scale (BPRS) ${ }^{42}$ and the Global Assessment of Functioning (GAF), respectively. Clinical information was obtained from the clinical psychiatrist in charge of each patient and was based on detailed clinical observations during hospitalization and/or long-term follow-up appointments during outpatient treatment. Diagnoses and clinical assessments were verified by two research psychiatrists who were blind to the ASSR data. None of the patients had a history of auditory disorders, neurological disorders, head trauma, electroconvulsive therapy, or substance/alcohol abuse. However, all patients were administered antipsychotics. Fifteen patients with schizophrenia were using new generation antipsychotics only, and 23 patients with schizophrenia were using conventional antipsychotics or a combination of conventional and new generation antipsychotics. The clinical variables of the patients were as follows: illness duration, $22.8 \pm 12.0$ years; chlorpromazine-equivalent antipsychotic dose, $945.1 \pm 896.5 \mathrm{mg} /$ day; and GAF score, $34.1 \pm 16.1$. The BPRS item scores were categorized into a four-dimensional model according to a previous report ${ }^{43}$ :

(1) Thinking disturbance (hallucinatory behavior, unusual thought content, and conceptual disorganization): $6.1 \pm 4.2$.

(2) Withdrawal/retardation (emotional withdrawal, blunted affect, and motor retardation): 5.1 \pm 4.0 .

(3) Hostile/suspiciousness (hostility, suspiciousness, and uncooperativeness): $2.8 \pm 2.9$.

(4) Anxious/depression (anxiety, guilt feelings, and depressive mood): $2.9 \pm 2.3$.

The subsequent report confirms that Thinking Disturbance and Withdrawal-Retardation reflect positive and negative symptoms, respectively ${ }^{44}$. The healthy subjects had no history of psychiatric, neurological, or auditory disorders. This study was approved by the Ethics Committee of the Kindai University Faculty of Medicine and was carried out in accordance with the ethical principles of the Declaration of Helsinki and its subsequent amendments. A complete description of the study was provided to each study participant, and written informed consent was obtained from all of the participants.

ASSR measurements. The ASSR was performed with a medical ERA device, Audera, following established technical protocol with minor modifications. The Audera device usually tests one ear at a time with TIP50 insert phones (Grason-Stader Inc.). In this study, the single tube from the insert phone for the left ear was replaced with a bifurcated tube so as to allow for the binaural presentation of tones. The bifurcated tube was placed so as not to touch the body or the clothing to avoid any interfering noises. The EEG was sampled from an electrode placed on the forehead around the middle point between the Fz and Fpz of the International 10-20 system. The electrodes on the left earlobe and the low forehead around the Fpz served as the reference and the ground electrodes, respectively. The electrode impedances were $<5 \mathrm{k} \Omega$. The $40-\mathrm{Hz}$ ASSR potentials were evoked by two kinds of auditory stimuli with intensity levels at $70 \mathrm{dBHL}$. The two stimuli used in this study are continuous sine wave tones with a carrier frequency $(\mathrm{CF})$ of $1,000 \mathrm{~Hz}$ that have either mixed modulation $(100 \% \mathrm{AM}$ plus $10 \% \mathrm{FM})$ at $46 \mathrm{~Hz}$, with which a sinusoidal change of the tone volume between 0 and $70 \mathrm{~dB}$ and a fluctuation of the CF tone between 900 and $1,100 \mathrm{~Hz}$ occur at the rate of $46 \mathrm{~Hz}$, or $100 \%$ AM, with which the tone volume sinusoidally changes between 0 and $70 \mathrm{~dB}$ at the rate of $40 \mathrm{~Hz}$. The $46-\mathrm{Hz}$ AM-FM ASSR is the test setting in Audera that is used for awake adults, whereas the 40-Hz AM ASSR is one of the basic settings that has been used, although via discrete tones, in prior ASSR studies on schizophrenia ${ }^{27,32}$. The 46-Hz AM-FM ASSR was slightly modulated from the $40-\mathrm{Hz}$ AM ASSR to increase the ASSR potentials via the addition of frequency modulation $^{45}$. The $46-\mathrm{Hz}$ AM-FM ASSR and the $40-\mathrm{Hz}$ AM ASSR were measured thrice for each subject in a counterbalanced manner. The subjects were instructed to sit and relax on a chair, keep their eyes closed, and 
remain motionless during ASSR measurements to avoid muscular artifact generation. The subjects were asked to open their eyes during a brief break between the three measurements to avoid falling asleep during any subsequent measurement.

Data acquisition. The presence or absence of the phase-locked response was automatically determined by a statistical algorithm adopted in the Audera system. Although the details of the algorithm are not available in the public domain, the following steps are implemented in the system. First, after eliminating the initial EEG sampling for approximately $10 \mathrm{~s}$ as an acclimation period, the system serially segments the continuous EEG sample during the tone stimulation into an epoch around every $0.1 \mathrm{~s}$. Every ten epochs are then overlaid as trials. Each trial is analyzed using a fast Fourier transform to calculate the amplitude and phase in the frequency domain corresponding to the modulation frequency. These outcomes are reported as vectors (Fig. 1). Moreover, the statistical algorithm applied phase coherence squared $\left(\mathrm{PC}^{2}\right)$ to estimate the phase coherence between the trials and the modulation frequency. The $\mathrm{PC}^{2}$ value was statistically evaluated by means of a circular variance to test for a $97 \%$ confidence criterion, resulting in phase-locked response detection. The $\mathrm{PC}^{2}$ value was updated during the measurement each time a new trial was added, and the system algorithm automatically terminated the stimulation and the data sampling when a phase-locked response was detected or when a phase-locked response could not be reasonably detected within 64 trials. In this study, the latter case was defined as a non-phaselocked response. The minimum number of trials to achieve a phase-locked response is 16, which represents the evaluation of 160 sampling epochs (10 epochs/trial times 16 trials). The maximum number of trials is 64 , which resulted in the evaluation of 640 sampling epochs (10 epochs/trial times 64 trials). The time taken to run one measurement ranges from 31 to $98 \mathrm{~s}$, according to the number of trials performed. To support the evaluation of the $40-\mathrm{Hz}$ gamma oscillations in the presence or absence of a phase-locked response, additional parameters were selected: (1) the number of trials to achieve a phase-locked response, which was displayed as the number of vectors in Fig. 1, and (2) the response amplitude (root mean square voltage; in microvolts) of the ASSR, which was calculated as the mean length of the vectors. The non-phase-locked response, which was judged not to phaselock within 64 trials, was assigned the maximum trial number of 64 in the statistical analysis of the number of trials required to achieve a phase-locked response. The results of three measurements for each parameter were averaged for each of the stimuli for each subject.

Statistics. The occurrence of the non-phase-locked response was compared between patients with schizophrenia and healthy subjects using Fisher's exact test. The clinical variables were compared between the all phase-locked and non-phase-locked groups of patients using binary logistic regression. The numbers of trials required to achieve the phase-locked response and the response amplitude were compared between the two groups via unpaired $t$-tests. The test-retest reliabilities for the ASSR measurements were examined across the entire sample of patients and healthy subjects using $\mathrm{ICCs}^{46,47}$ in terms of the number of trials required to achieve the phase-locked response and the response amplitude. A one-way random, single-measure ICC analysis and a one-way random, average-measure ICC analysis were performed to examine the reliabilities of single and triple measurements of ASSR, respectively. According to a previous report ${ }^{48}$, the quality of ICC was judged as follows; ICC $<0.00$, poor; 0.00 to 0.20 , slight; 0.21 to 0.40 , fair; 0.41 to 0.60 , moderate; 0.61 to 0.80 , substantial; $0.81-1.00$ almost perfect. All statistical tests were two-tailed, and the threshold for the significance of $P$ values was set at 0.05. SPSS version 25.0 (IBM Inc., Armonk, NY, USA) was used for the statistical analyses.

\section{Data availability}

The data supporting the findings of this study are available from the corresponding author upon reasonable request.

Received: 4 August 2021; Accepted: 17 December 2021

Published online: 07 January 2022

\section{References}

1. Lewis, D. A., Hashimoto, T. \& Volk, D. W. Cortical inhibitory neurons and schizophrenia. Nat. Rev. Neurosci. 6, 312-324. https:// doi.org/10.1038/nrn1648 (2005).

2. Uhlhaas, P. J. \& Singer, W. Abnormal neural oscillations and synchrony in schizophrenia. Nat. Rev. Neurosci. 11, 100-113. https:// doi.org/10.1038/nrn2774 (2010).

3. Javitt, D. C., Spencer, K. M., Thaker, G. K., Winterer, G. \& Hajos, M. Neurophysiological biomarkers for drug development in schizophrenia. Nat. Rev. Drug Discov. 7, 68-83. https://doi.org/10.1038/nrd2463 (2008).

4. McNally, J. M. \& McCarley, R. W. Gamma band oscillations: a key to understanding schizophrenia symptoms and neural circuit abnormalities. Curr. Opin. Psychiatry 29, 202-210. https://doi.org/10.1097/YCO.0000000000000244 (2016).

5. Senkowski, D. \& Gallinat, J. Dysfunctional prefrontal gamma-band oscillations reflect working memory and other cognitive deficits in schizophrenia. Biol. Psychiatry 77, 1010-1019. https://doi.org/10.1016/j.biopsych.2015.02.034 (2015).

6. Sun, Y. et al. gamma oscillations in schizophrenia: mechanisms and clinical significance. Brain Res. 1413, 98-114. https://doi.org/ 10.1016/j.brainres.2011.06.065 (2011).

7. Gandal, M. J., Edgar, J. C., Klook, K. \& Siegel, S. J. Gamma synchrony: towards a translational biomarker for the treatment-resistant symptoms of schizophrenia. Neuropharmacology 62, 1504-1518. https://doi.org/10.1016/j.neuropharm.2011.02.007 (2012).

8. Uhlhaas, P. J., Haenschel, C., Nikolic, D. \& Singer, W. The role of oscillations and synchrony in cortical networks and their putative relevance for the pathophysiology of schizophrenia. Schizophr Bull. 34, 927-943. https://doi.org/10.1093/schbul/sbn062 (2008)

9. Galambos, R., Makeig, S. \& Talmachoff, P. J. A 40-Hz auditory potential recorded from the human scalp. Proc. Natl. Acad. Sci. U S A 78, 2643-2647. https://doi.org/10.1073/pnas.78.4.2643 (1981).

10. Thune, H., Recasens, M. \& Uhlhaas, P. J. The $40-\mathrm{Hz}$ auditory steady-state response in patients with schizophrenia: a meta-analysis. JAMA Psychiat. 73, 1145-1153. https://doi.org/10.1001/jamapsychiatry.2016.2619 (2016). 
11. Gonzalez-Burgos, G. \& Lewis, D. A. NMDA receptor hypofunction, parvalbumin-positive neurons, and cortical gamma oscillations in schizophrenia. Schizophr Bull. 38, 950-957. https://doi.org/10.1093/schbul/sbs010 (2012).

12. Jadi, M. P., Behrens, M. M. \& Sejnowski, T. J. Abnormal gamma oscillations in N-methyl-D-aspartate receptor hypofunction models of Schizophrenia. Biol. Psychiatry 79, 716-726. https://doi.org/10.1016/j.biopsych.2015.07.005 (2016).

13. Tada, M. et al. Gamma-band auditory steady-state response as a neurophysiological marker for excitation and inhibition balance: a review for understanding schizophrenia and other neuropsychiatric disorders. Clin. EEG Neurosci. 51, 234-243. https://doi.org/ $10.1177 / 1550059419868872(2020)$.

14. Cardin, J. A. et al. Driving fast-spiking cells induces gamma rhythm and controls sensory responses. Nature 459, 663-667. https:// doi.org/10.1038/nature08002 (2009).

15. Sohal, V. S., Zhang, F., Yizhar, O. \& Deisseroth, K. Parvalbumin neurons and gamma rhythms enhance cortical circuit performance. Nature 459, 698-702. https://doi.org/10.1038/nature07991 (2009).

16. Lewis, D. A. \& Gonzalez-Burgos, G. Pathophysiologically based treatment interventions in schizophrenia. Nat. Med. 12, 1016-1022. https://doi.org/10.1038/nm1478 (2006).

17. Gonzalez-Burgos, G., Cho, R. Y. \& Lewis, D. A. Alterations in cortical network oscillations and parvalbumin neurons in schizophrenia. Biol. Psychiatry 77, 1031-1040. https://doi.org/10.1016/j.biopsych.2015.03.010 (2015).

18. Wassef, A., Baker, J. \& Kochan, L. D. GABA and schizophrenia: a review of basic science and clinical studies. J. Clin. Psychopharmacol. 23, 601-640. https://doi.org/10.1097/01.jcp.0000095349.32154.a5 (2003).

19. Lewis, D. A. et al. Subunit-selective modulation of GABA type A receptor neurotransmission and cognition in schizophrenia. Am. J. Psychiatry 165, 1585-1593. https://doi.org/10.1176/appi.ajp.2008.08030395 (2008).

20. Buchanan, R. W. et al. A randomized clinical trial of MK-0777 for the treatment of cognitive impairments in people with schizophrenia. Biol. Psychiatry 69, 442-449. https://doi.org/10.1016/j.biopsych.2010.09.052 (2011).

21. Rudy, B. \& McBain, C. J. Kv3 channels: voltage-gated K+ channels designed for high-frequency repetitive firing. Trends Neurosci. 24, 517-526. https://doi.org/10.1016/s0166-2236(00)01892-0 (2001).

22. Rosato-Siri, M. D. et al. A novel modulator of Kv3 potassium channels regulates the firing of parvalbumin-positive cortical interneurons. J. Pharmacol. Exp. Ther. 354, 251-260. https://doi.org/10.1124/jpet.115.225748 (2015).

23. Yanagi, M. et al. Kv3.1-containing $\mathrm{K}(+)$ channels are reduced in untreated schizophrenia and normalized with antipsychotic drugs. Mol. Psychiatry 19, 573-579. https://doi.org/10.1038/mp.2013.49 (2014).

24. Light, G. A. et al. Neurophysiological biomarkers for schizophrenia therapeutics. Biomarkers Neuropsychiatry https://doi.org/10. 1016/j.bionps.2020.100012 (2020).

25. Clementz, B. A. et al. Identification of distinct psychosis biotypes using brain-based biomarkers. Am. J. Psychiatry 173, 373-384. https://doi.org/10.1176/appi.ajp.2015.14091200 (2016).

26. Hong, L. E. et al. Evoked gamma band synchronization and the liability for schizophrenia. Schizophr. Res. 70, 293-302. https:// doi.org/10.1016/j.schres.2003.12.011 (2004).

27. Krishnan, G. P. et al. Steady state and induced auditory gamma deficits in schizophrenia. Neuroimage 47, 1711-1719. https://doi. org/10.1016/j.neuroimage.2009.03.085 (2009).

28. Parthasarathy, A., Hancock, K. E., Bennett, K., DeGruttola, V. \& Polley, D. B. Bottom-up and top-down neural signatures of disordered multi-talker speech perception in adults with normal hearing. Elife https://doi.org/10.7554/eLife.51419 (2020).

29. Brown, M. \& Kuperberg, G. R. A hierarchical generative framework of language processing: linking language perception, interpretation, and production abnormalities in schizophrenia. Front. Hum. Neurosci. 9, 643. https://doi.org/10.3389/fnhum.2015.00643 (2015).

30. Hugdahl, K. \& Sommer, I. E. Auditory verbal hallucinations in schizophrenia from a levels of explanation perspective. Schizophr. Bull. 44, 234-241. https://doi.org/10.1093/schbul/sbx142 (2018).

31. Hirano, Y. et al. Spontaneous gamma activity in schizophrenia. JAMA Psychiat. 72, 813-821. https://doi.org/10.1001/jamapsychi atry.2014.2642 (2015).

32. Teale, P. et al. Cortical source estimates of gamma band amplitude and phase are different in schizophrenia. Neuroimage 42, 1481-1489. https://doi.org/10.1016/j.neuroimage.2008.06.020 (2008).

33. Roach, B. J. \& Mathalon, D. H. Event-related EEG time-frequency analysis: an overview of measures and an analysis of early gamma band phase locking in schizophrenia. Schizophr. Bull. 34, 907-926. https://doi.org/10.1093/schbul/sbn093 (2008).

34. Parker, D. A. et al. Auditory steady-state EEG response across the schizo-bipolar spectrum. Schizophr. Res. 209, 218-226. https:// doi.org/10.1016/j.schres.2019.04.014 (2019).

35. Light, G. A. et al. Gamma band oscillations reveal neural network cortical coherence dysfunction in schizophrenia patients. Biol. Psychiatry 60, 1231-1240. https://doi.org/10.1016/j.biopsych.2006.03.055 (2006).

36. Tada, M. et al. Differential alterations of auditory gamma oscillatory responses between pre-onset high-risk individuals and firstepisode schizophrenia. Cereb. Cortex 26, 1027-1035. https://doi.org/10.1093/cercor/bhu278 (2016).

37. Griskova-Bulanova, I., Hubl, D., van Swam, C., Dierks, T. \& Koenig, T. Early- and late-latency gamma auditory steady-state response in schizophrenia during closed eyes: does hallucination status matter? Clin. Neurophysiol. 127, 2214-2221. https://doi. org/10.1016/j.clinph.2016.02.009 (2016).

38. Koshiyama, D. et al. Auditory gamma oscillations predict global symptomatic outcome in the early stages of psychosis: a longitudinal investigation. Clin. Neurophysiol. 129, 2268-2275. https://doi.org/10.1016/j.clinph.2018.08.007 (2018).

39. Picton, T. W., John, M. S., Purcell, D. W. \& Plourde, G. Human auditory steady-state responses: the effects of recording technique and state of arousal. Anesth. Analg. 97, 1396-1402. https://doi.org/10.1213/01.ane.0000082994.22466.dd (2003).

40. Ivleva, E. I. et al. Gray matter volume as an intermediate phenotype for psychosis: bipolar-schizophrenia network on intermediate phenotypes (B-SNIP). Am. J. Psychiatry 170, 1285-1296. https://doi.org/10.1176/appi.ajp.2013.13010126 (2013).

41. American Psychiatric Association. DSM-5 Task Force. Diagnostic and statistical manual of mental disorders: DSM-5. 5th edn, (American Psychiatric Association, 2013).

42. Kolakowska, T. Brief Psychiatric Rating Scale: Glossaries and Rating Instructions (Oxford University, 1976).

43. Overall, J. E., Hollister, L. E. \& Pichot, P. Major psychiatric disorders. A four-dimensional model. Arch. Gen. Psychiatry 16, 146-151. https://doi.org/10.1001/archpsyc.1967.01730200014003 (1967).

44. Nicholson, I. R., Chapman, J. E. \& Neufeld, R. W. Variability in BPRS definitions of positive and negative symptoms. Schizophr. Res. 17, 177-185. https://doi.org/10.1016/0920-9964(94)00088-p (1995).

45. Cohen, L. T., Rickards, F. W. \& Clark, G. M. A comparison of steady-state evoked potentials to modulated tones in awake and sleeping humans. J. Acoust Soc. Am. 90, 2467-2479. https://doi.org/10.1121/1.402050 (1991).

46. Hirano, Y., Nakamura, I., Tamura, S. \& Onitsuka, T. Long-term test-retest reliability of auditory gamma oscillations between different clinical EEG systems. Front. Psychiatry 11, 876. https://doi.org/10.3389/fpsyt.2020.00876 (2020).

47. Shrout, P. E. \& Fleiss, J. L. Intraclass correlations: uses in assessing rater reliability. Psychol. Bull. 86, 420-428. https://doi.org/10. 1037//0033-2909.86.2.420 (1979).

48. Landis, J. R. \& Koch, G. G. The measurement of observer agreement for categorical data. Biometrics 33, 159-174 (1977). 


\section{Acknowledgements}

This work was supported by a Grant-in-Aid for Scientific Research from the Japan Society for the Promotion of Science (grant number 16K10229 to O.S. and 17K10320 to M.Y.). All authors report no financial relationships with commercial interests.

We appreciate and thank the volunteers who participated in this study and Ms. Shizuka Ishida for the technical support to measure ASSR and for data analysis. Enago (www.enago.jp) is also thanked for the English language review.

\section{Author contributions}

M.Y. designed the study. M.Y. and F.H. collected the ASSR data. A.T. and S.O. obtained the clinical data of patients with schizophrenia. M.Y. managed the analyses. M.Y. wrote the manuscript. O.S. interpreted the data and supervised this study. All authors contributed to and approved the final manuscript.

\section{Competing interests}

The authors declare no competing interests.

\section{Additional information}

Supplementary Information The online version contains supplementary material available at https://doi.org/ 10.1038/s41598-021-04278-5.

Correspondence and requests for materials should be addressed to M.Y.

Reprints and permissions information is available at www.nature.com/reprints.

Publisher's note Springer Nature remains neutral with regard to jurisdictional claims in published maps and institutional affiliations.

(c) Open Access This article is licensed under a Creative Commons Attribution 4.0 International License, which permits use, sharing, adaptation, distribution and reproduction in any medium or format, as long as you give appropriate credit to the original author(s) and the source, provide a link to the Creative Commons licence, and indicate if changes were made. The images or other third party material in this article are included in the article's Creative Commons licence, unless indicated otherwise in a credit line to the material. If material is not included in the article's Creative Commons licence and your intended use is not permitted by statutory regulation or exceeds the permitted use, you will need to obtain permission directly from the copyright holder. To view a copy of this licence, visit http://creativecommons.org/licenses/by/4.0/.

(c) The Author(s) 2022 